JOURNAL OF SYNCHROTRON RADIATION

ISSN 1600-5775

Received 4 December 2020

Accepted 13 January 2021

Edited by M. Yamamoto, RIKEN SPring-8 Center, Japan

Keywords: undulator; field harmonics; radiation harmonics; free-electron laser.

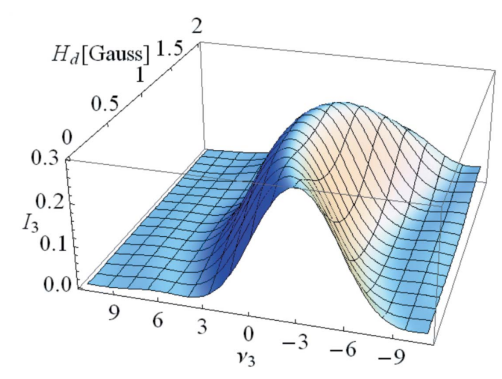

C 2021 International Union of Crystallography

\section{Theoretical spectral analysis of FEL radiation from multi-harmonic undulators. Corrigendum}

\author{
K. Zhukovsky*
}

Department of Theoretical Physics, Faculty of Physics, M. V. Lomonosov Moscow State University, Moscow 119991, Russian Federation. *Correspondence e-mail: zhukovsk@physics.msu.ru

A correction to an equation in the paper by Zhukovsky [(2020). J. Synchrotron Rad. 27, 1648-1661] is made and, following from this, values of some Bessel coefficients and some harmonic intensities from an elliptic undulator with the third field harmonic.

The author would like to correct a misprint in formula (3) of Zhukovsky (2020) and communicate the change of the numerical values in equations (23), (24) and in Fig. 9.

The argument of the generalized Bessel function (3) in Zhukovsky (2020) for the magnetic field

$$
\begin{gathered}
\mathbf{H}=H_{0}\left[\sin \left(k_{\lambda} z\right)+d \sin \left(p k_{\lambda} z\right), d_{1} \sin \left(h k_{\lambda} z\right)+d_{2} \cos \left(l k_{\lambda} z\right), 0\right] \\
k_{\lambda}=2 \pi / \lambda_{\mathrm{u}, x}, \quad d, d_{1}, d_{2} \in \text { real, } h, l, p \in \text { integers }
\end{gathered}
$$

was erroneously written in equation (3) of Zhukovsky (2020) as a cosine instead of an exponential; formula (3) should correctly read as follows,

$$
\begin{aligned}
J_{n}^{m}\left(\xi_{i}\right)= & \int_{0}^{2 \pi} \frac{\mathrm{d} \alpha}{2 \pi} \exp \left(i \left\{n \alpha+\xi_{1} \sin (h \alpha)+\xi_{2} \cos (l \alpha)\right.\right. \\
& +\xi_{3} \sin \alpha+\xi_{4} \sin (2 \alpha)+\xi_{5} \sin (2 h \alpha) \\
& +\xi_{6} \sin (2 l \alpha)+\xi_{7} \cos [(l+h) \alpha] \\
& +\xi_{8} \cos [(l-h) \alpha]+\xi_{0} \sin (p \alpha) \\
& +\xi_{9} \sin [(p+1) \alpha]+\xi_{10} \sin [(p-1) \alpha] \\
& \left.\left.+\xi_{11} \sin (2 p \alpha)\right\}\right)
\end{aligned}
$$

The undulator radiation (UR) harmonic powers in the undulator with $N=30$ periods, $k=2.216$ and period $\lambda_{\mathrm{u}}=2.3 \mathrm{~cm}$, and the beam with energy spread $\sigma_{\mathrm{e}}=0.1 \%$, are shown here in the revised versions of Figures $9(a)$ and $9(b)$, which replace the respective figures in the original paper. The ratio between the harmonic intensity of the undulator and the magnetic field (21) [see Zhukovsky (2020)] has somewhat changed, but, for the studied undulator field (22) in Zhukovsky (2020),

$$
\mathbf{H}=H_{0}\left[\sin \left(k_{\lambda} z\right), 0.25 \cos \left(k_{\lambda} z\right)+\sin \left(3 k_{\lambda} z\right), 0\right]
$$

the third UR harmonic remains strong in both polarizations and the change of the Bessel coefficients for the third harmonic, $f_{n=3}$, is minor as compared with their values in Zhukovsky (2020). New values, reported below, substitute those in formulae (23) and (24) of Zhukovsky (2020), 

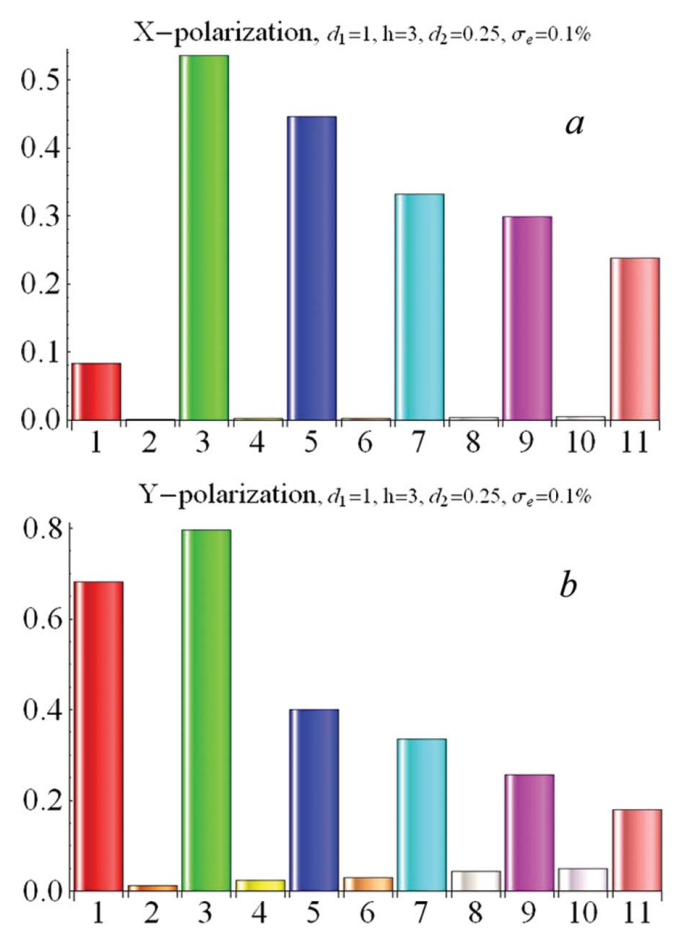

Figure 9

(a) $x$-polarization and (b) $y$-polarization of the on-axis UR harmonics intensity (in relative units) for the elliptic undulator with period $\lambda_{\mathrm{u}}=$ $2.3 \mathrm{~cm}, k=2.21622, h=3, d_{1}=1, d_{2}=+0.25, l=1, \sigma_{\mathrm{e}}=10^{-3}, N=30$.

$$
\begin{gathered}
f_{x ; n=1 \ldots 9}=\left\{\begin{array}{c}
0.290,0.007,0.256,0.010,0.151, \\
0.009,0.101,0.009,0.081\}
\end{array}\right. \\
f_{y ; n=1 \ldots 9}=\{0.830,0.056,0.313,0.042,0.143, \\
0.033,0.102,0.033,0.075\} .
\end{gathered}
$$

The changes of the numerical values in (26), (27), (29), (30) are minor and we omit them for brevity. The harmonic intensities of the UR spectrum, obtained with formula (3) for the undulator in Zhukovsky (2020), are now revised and reported in the revised Figures $9(a)$ and $9(b)$ for $x$ - and $y$ polarizations, respectively. We consider [see also Zhukovsky (2020)] an undulator with period $\lambda_{\mathrm{u}}=2.3 \mathrm{~cm}, k=2.216$, magnetic field given by (22), electron beam relativistic parameter $\gamma=12.72$, emittances $\varepsilon_{x}=1.5 \mathrm{~mm} \mathrm{mrad}$ and $\varepsilon_{y}=$ $0.35 \mathrm{~mm}$ mrad, Twiss parameters $\beta_{x}=43.66 \mathrm{~cm}$ and $\beta_{y}=$ $28.75 \mathrm{~cm}$, beam sections $\sigma_{x}=809 \mu \mathrm{m}$ and $\sigma_{y}=317 \mu \mathrm{m}$, divergences $\theta_{x}=4.5 \mathrm{mrad}$ and $\theta_{y}=1.6 \mathrm{mrad}$ and electron energy spread $\sigma_{\mathrm{e}}=0.1 \%$. The corrections have not affected other calculations, results and conclusions in Zhukovsky (2020). The author apologizes for the inconvenience.

\section{References}

Zhukovsky, K. (2020). J. Synchrotron Rad. 27, 1648-1661. 